\title{
Power Aware Routing Protocol (PARP) for Wireless Sensor Networks
}

\author{
R. Prema ${ }^{1}$, R. Rangarajan ${ }^{2}$ \\ ${ }^{1}$ Department of Electronics, Karpagam University, Coimbatore, India \\ ${ }^{2}$ V.S.B. Engineering College, Karur, India \\ Email: rpremaphd@yahoo.com
}

Received February 5, 2012; revised March 16, 2012; accepted April 7, 2012

\begin{abstract}
Several wireless sensor network applications ought to decide the intrinsic variance between energy efficient communication and the requirement to attain preferred quality of service (QoS) such as packet delivery ratio, delay and to reduce the power consumption of wireless sensor nodes. In order to address this challenge, we propose the Power Aware Routing Protocol (PARP), which attains application-specified communication delays at low energy cost by dynamically adapting transmission power and routing decisions. Extensive simulation results prove that the proposed PARP attains better QoS and reduced power consumption.
\end{abstract}

Keywords: Power; Routing; QoS; Sensor Networks

\section{Introduction}

Smart environments represent the next evolutionary development step in building, utilities, industrial, home, shipboard, and transportation systems automation. Like any sentient organism, the smart environment relies first and foremost on sensory data from the real world. Sensory data comes from multiple sensors of different modalities in distributed locations. The smart environment needs information about its surroundings as well as about its internal workings; this is captured in biological systems by the distinction between exteroceptors and proprioceptors.

The challenges in the hierarchy of: detecting the relevant quantities, monitoring and collecting the data, assessing and evaluating the information, formulating meaningful user displays, and performing decision-making and alarm functions are enormous. The information needed by smart environments is provided by Distributed Wireless Sensor Networks, which are responsible for sensing as well as for the first stages of the processing hierarchy. The importance of sensor networks is highlighted by the number of recent funding initiatives, including the DARPA SENSIT program, military programs, and NSF Program Announcements. Desirable functions for sensor nodes include: ease of installation, self-identification, self-diagnosis, reliability, time awareness for coordination with other nodes, some software functions and DSP, and standard control protocols and network interfaces.

\section{Literature Review}

Power-aware algorithms for routing in WSNs have received considerable attention over the past few years. A distributed position-based algorithm to form topologies containing a minimum total energy route between any pair of connected nodes is proposed in [1]. Based on this initial work, a computationally simpler protocol with better performance is described in [2]. Similar topology control algorithms based on discretization of the coverage region of a node into cones are proposed in $[3,4]$. The idea is to select appropriate transmitter power levels to guarantee network connectivity while at the same time transmission energy is saved.

Putting a node into sleep mode whenever its active collaboration in the current network task is not required is another way to save energy. The geographical adaptive fidelity (GAF) algorithm [5] conserves energy by turning off nodes that are equivalent from a routing perspective, thereby keeping a constant level of routing fidelity. An improvement of GAF based on a relationship between optimal transmission range and traffic is described [6]. In Span [7], the decision whether a node should be awake or sleep is made depending on how many of its neighbors will get benefit and how much remaining energy it has. The sparse topology and energy management (STEM) protocol [8] puts nodes aggressively into sleep mode and only wakes them up when they are needed to forward data. Data fusion is a technique that can be used to reduce the amount of redundant information prevalent in 
dense sensor networks. By combining data with equal semantics, unnecessary power consumption due to transmission and processing of duplicate data is prevented. Two prominent routing protocols that use upper layer information for data fusion as well as making routing decisions are Directed Diffusion [9] and SPIN [10]. Application-specific fusion enables even more sophisticated data and node management functionalities insideWSNs [11]. Both sleep scheduling and data fusion are desirable functionalities which may complement energy-efficient MAC androuting protocols.

The scalability problem of WSN protocols is discussed in [12]. The authors argue that localized algorithms, where a node exchanges information only with its direct neighbors, provide for good scalability. Our proposed routing algorithms are localized in the sense that each node decides on the next hop based only on the position of itself, of its neighbors, and possibly of the destination node. Other techniques developed to cope with scalability in large sensor networks are to introduce heterogeneity [13], hierarchy [14-16], clustering [17-19], and location-awareness [5,20,21].

\section{Power Aware Routing Protocol (PARP) for Wireless Sensor Networks}

\subsection{Estimation of Link Quality}

The communication in mobile ad-hoc network is based on electronic signals. In mobile ad-hoc networks it is possible that a communication path (route) will break. This will happen primarily because of the nodes present in the network are moving around the region. The Figure $\mathbf{1}$, depicts the scenario when the link is active. In the Figure 1, three nodes are present namely a, b and c. The node-b is within the range of the node-a and node-c. But, the node-a is not within the range of node-c and node-c is not within the range of node-a. Hence for transmission of data from node-a to node-c, the node-b acts as an intermediate node. After certain duration, due to the mobility of sensor nodes, the link gets break and the data communication between the nodes becomes unreliable.

Due to the mobility of nodes present in wireless sensor network it becomes mandatory to consider the quality of the link.

To be able to see that when a node in the wireless sensor network is moving and hence a route is about to break which is shown in Figure 2. So that factor, it is probable to measure the quality of the signal and based upon that presumption, when the link is going to break. This information which is identified by the physical layer is send to the upper layer when packets are received from a node, and then indicate that node is in pre-emptive zone. Pre-emptive zone is the region where the signal strength is weaker which leads to the link failure. Pre-emptive

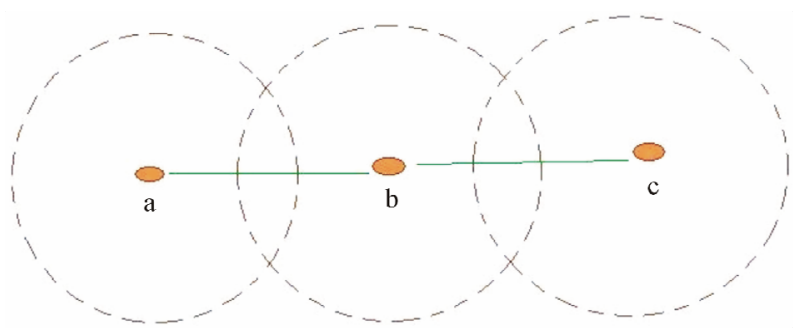

Figure 1. Before the link breaks.

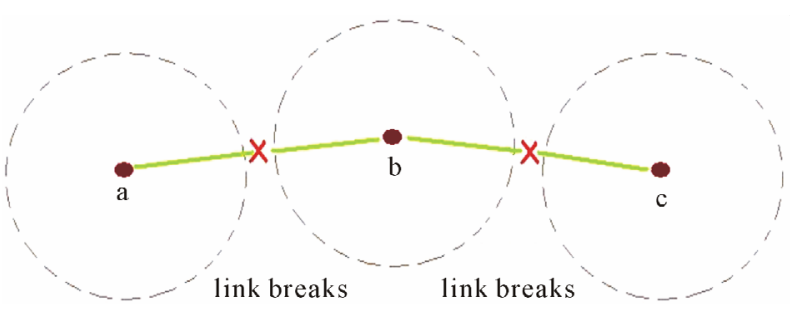

Figure 2. After the link breaks.

zone uses the pre-emptive threshold value to fix the preemptive zone's location. Thus, using the received signal strength from physical layer, the quality of the link is predicted and then the links which are having low signal strength will be discarded from the route selection.

When a sending node broadcasts RTS packet, it piggybacks its transmission power. While receiving the RTS packet, the projected node quantifies the strength of the signal received.

$$
P_{R}=P_{T}\left(\lambda / 4 \prod d\right)^{2} *\left(U G_{T}\right) *\left(U G_{R}\right)
$$

Hence,

$$
L_{q}=P_{R}
$$

where,

$P_{R}$ refers Power of the Receiving node,

$P_{T}$ stands for Power of the Transmitting node,

$\lambda$ stands for wavelength carrier,

$d$ is the distance between the sending and the receiving node,

$U G_{R}$ stands for unity gain of receiving omni-directional antenna,

$U G_{T}$ stands for unity gain of transmitting omni-directional antenna.

$$
R N=\max \left(L_{q} \& R_{P O W}\right)
$$

where,

$\mathrm{CV}=$ Cost Value,

$L_{q}=$ Link quality,

$R_{P O W}=$ Residual Power of the sensor node.

In the proposed work Power Aware Routing Protocol (PARP) a cost value (CV) is calculated. CV is computed based on the on the quality of the link of each wireless sensor node. Among all the sensor nodes in the network, there are some robust nodes. These robust nodes serve as 
the backbone for the routing in wireless sensor networks. The remaining sensor nodes are common sensor nodes. Each robust node maintains a table of sensor node power at other robust nodes. So in the route, each robust node will compute the end-to-end power from itself to any other robust nodes. The sensor node power is estimated and updated periodically by each robust node. The robust node which is nearest to the source node finds the robust nodes which are along the route towards destination sensor node. Then packets will be forwarded through these robust nodes to the destination node. Since robust nodes have better communication capability than common nodes, most of the time the power is less than the maximum power.

\subsection{Working Mechanism of PARP}

1) Each robust node can arrive at nearby robust nodes directly. When a robust node goes out of a grid, it initiates a robust node election process in the grid and a new robust node will be selected.

2) Each Robust node holds a table of node power. Each Robust node can calculate the end-to-end power from itself to any other robust nodes. The node delay is estimated and updated periodically by each robust node.

3) Incase a source node $S$ needs to setup a route to a destination D. It is considered by the case where the source node $\mathrm{S}$ itself is a robust node. In this case, first the robust node $\mathrm{S}$ needs to know about the current location of the destination node D. With the information of D's location, S knows about the grid Ld where D stays, and the Robust node Ltd in the grid Ld.

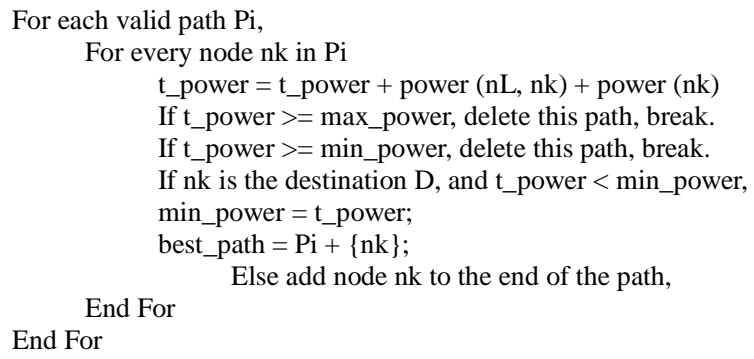

Pseudo code for Robust Sensor node election

4) Then $S$ calculates the minimum power between $S$ and Ltd by means of the power table, and also discovers the route with the minimum power. If the minimum power is greater than the required power, then the route can not be established. The source sensor node generates a unique req_id for each route request. When an intermediate node obtains the REQ packet, it adds the powers of the incoming link and itself to t_power, and compares the updated t_power with the max_power. If t_power is less than the max_power, it adds up itself to the route_list, and forwards the REQ packet to the neighbors. If t_power is greater than max_power, the node will drop the REQ packet.

5) If the minimum power between $S$ and Ltd is less than the maximum power, sensor node $S$ will notify Ltd to locate a route to the destination D. Then Ltd will update the t_power by adding the power between Ltd and D. If the updated t_power is less than max_power, a valid route is found. Ltd will send an ACK (acknowledge) packet to $\mathrm{S}$ along the reverse path to ascertain that the route is setup. And each node in the route will updates its node power. After that $\mathrm{S}$ can start sending data.

6) If $S$ is not a Robust node, then $S$ will first discover a path to the nearby Robust node with less power than required. Node S sends out the route request (REQ) packet by flooding to all the sensor nodes in its grid. Only sensor nodes in the same grid will process and forward the REQ packet. When a node gets the REQ packet, it will update the power from source to their locations ( power). If t_power is less than max_power, it adds itself to the route_list, and forwards the REQ packet to the neighbors. If t_power is larger than max_power, the node will drop the REQ packet. When the Robust node in this grid gets the first REQ packet, it also updates the t_power and compares it with max_power. If t_power is less than max_power, it will calculate the minimum power between itself and the robust node which is nearest to the destination. The remaining steps are the same as above.

7) Sensor node power and current location information of robust nodes has to be updated and distributed among all robust nodes. The distribution is done periodically, and the length of the updating period depends on the network dynamics, such as sensor node mobility, sensor network traffic, sensor node communication capability, etc.

\subsection{Election of Robust Node}

At the start, one robust node is set in each grid. We need an election mechanism to produce new Robust nodes because robust nodes also move around. When a Robust node leaves its current grid or due to any other reason there is no robust node in the grid. Suppose, there are more Robust nodes in the current grid of the network, then, the next node with least weighted value from the sorted list will be chosen as the new Robust node for the grid. In the proposed routing algorithm, we need to compute the minimum delay between two robust nodes, and find the path with the minimum delay. This Power Aware Routing Protocol (PARP) results in reduced power consumption and delay as shown in Figures 3-4. It also increases packet delivery ratio which depicts in Figure 5. 


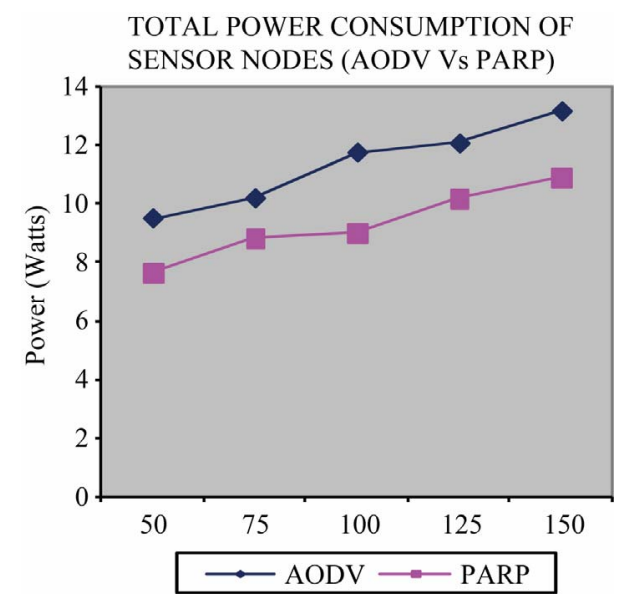

Figure 3. Pausetime vs. total power consumption.

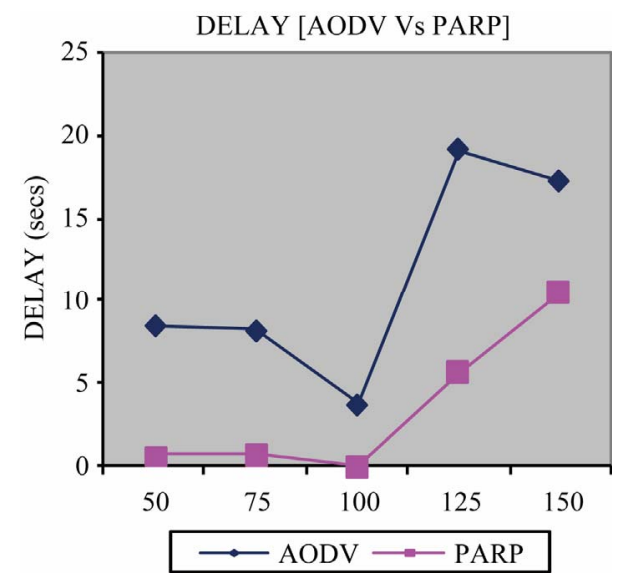

Figure 4. Pausetime vs. delay.

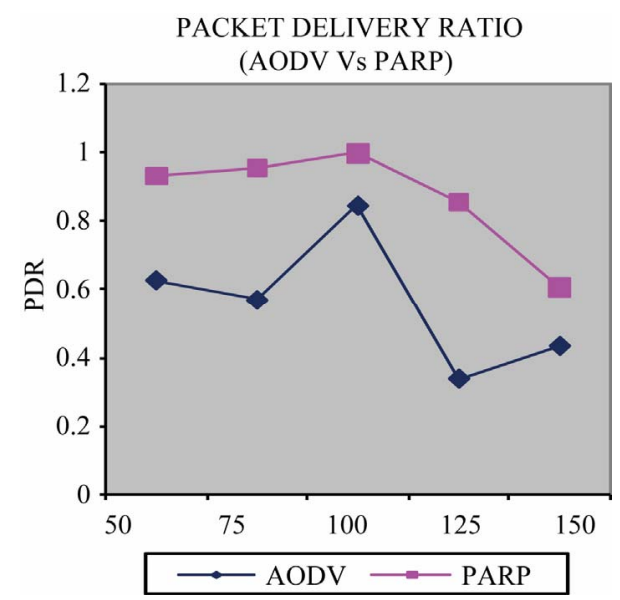

Figure 5. Pausetime vs. packet delivery ratio.

\section{Simulation Settings \& Graphs}

The simulation settings are shown in Table 1.

\section{Conclusion}

This paper proposed power aware routing protocol for
Table 1. Simulation settings.

\begin{tabular}{cc}
\hline No. of Nodes & $50,75,100,125$ and 150 \\
Area Size & $1000 \times 1000$ \\
Mac & 802.11 \\
Radio Range & $250 \mathrm{~m}$ \\
Simulation Time & $50 \mathrm{sec}$ \\
Traffic Source & CBR \\
Packet Size & $512 \mathrm{~KB}$ \\
Mobility Model & Random Way Point \\
Speed & $5 \mathrm{~m} / \mathrm{s}$ \\
Pause time & 100 Seconds \\
\hline
\end{tabular}

wireless sensor networks. PARP uses link quality estimation and power aware routing which results in reduced power consumption and delay with increased packet delivery ratio.

\section{REFERENCES}

[1] V. Rodoplu and T. H. Meng, "Minimum Energy Mobile Wireless Networks," IEEE Journal on Selected Areas in Communications, Vol. 17, No. 8, 1999, pp. 1333-1344. doi:10.1109/49.779917

[2] L. Li and J. Y. Halpern, "Minimum-Energy Mobile Wireless Networks Revisited," Proceedings of IEEE International Conference on Communications (ICC), 11-14 June 2001, pp. 278-283.

[3] L. Li, J. Y. Halpern, P. Bahl, Y.-M. Wang and R. Wattenhofer, "A Cone-Based Distributed Topology-Control Algorithm for Wireless Multi-Hop Networks,” IEEE/ ACM Transactions on Networking, Vol. 13, No. 1, 2005, pp. 147-159. doi:10.1109/TNET.2004.842229

[4] R. Wattenhofer, L. Li, P. Bahl and Y.-M. Wang, "Distributed Topology Control for Power Efficient Operation in Multihop Wireless Ad Hoc Networks," Proceedings of IEEE INFOCOM, Vol. 3, 2001, pp. 1388-1397.

[5] Y. Xu, J. Heidemann and D. Estrin, "Geography-Informed Energy Conservation for Ad Hoc Routing," Proceedings of ACM/IEEE International Conference on Mobile Computing and Networking (MobiCom), July 2001, pp. 70-84.

[6] Q. Gao, K. J. Blow, D. J. Holding, I. W. Marshall and X. Peng, "Routing Analysis and Energy Efficiency in Wireless Sensor Networks," Proceedings of IEEE International Symposium on Circuits and Systems (ISCAS 2004), Vol. 2, 31 May-2 June 2004, pp. 533-536.

[7] B. Chen, K. Jamieson, H. Balakrishnan and R. Morris. "Span: An Energy-Efficient Coordination Algorithm for Topology Maintenance in Ad Hoc Wireless Networks," Proceedings of ACM/IEEE International Conference on Mobile Computing and Networking (MobiCom), July 2001, pp. 85-96. 
[8] C. Schurgers, V. Tsiatsis, S. Ganeriwal and M. Srivastava, “Topology Management for Sensor Networks: Exploiting Latency and Density," Proceedings of ACM International Symposium on Mobile Ad Hoc Networking and Computing (MobiHoc), June 2002, pp. 135-145.

[9] C. Intanagonwiwat, R. Govindan and D. Estrin, "Directed diffusion: A Scalable and Robust Communication Paradigm for Sensor Networks," Proceedings of ACM/IEEE International Conference on Mobile Computing and Networking (MobiCom), August 2000, pp. 56-67.

[10] J. Kulik, W. Heinzelman and H. Balakrishnan, "Negotiation-Based Protocols for Disseminating Information in Wireless Sensor Networks," Wireless Networks, Vol. 8, No. 2-3, 2002, pp. 169-185. doi:10.1023/A:1013715909417

[11] W. Heinzelman, A. Chandrakasan and H. Balakrishnan, "Energy-Efficient Communication Protocol for Wireless Microsensor Networks," Proceedings of Hawaiian International Conference on Systems Science, 4-7 January 2000, pp. 1-10. doi:10.1109/HICSS.2000.926982

[12] D. Estrin, R. Govindan, J. Heidemann and S. Kumar, "Next Century Challenges: Scalable Coordination in Sensor Networks," Proceedings of ACM/IEEE International Conference on Mobile Computing and Networking (MobiCom), August 1999, pp. 263-270.

[13] E. J. Duarte-Melo and M. Liu, “Analysis of Energy Consumption and Lifetime of Heterogeneous Wireless Sensor Networks," Proceedings of IEEE GLOBCOM, 17-21 November 2002, pp. 21-25.

[14] J. Pan, L. Cai, Y. T. Hou, Y. Shi and S. X. Shen, "Optimal Base-Station Locations in Two-Tiered Wireless Sensor Networks," IEEE Transactions on Mobile Computing,
Vol. 4, No. 5, 2005, pp. 458-473. doi:10.1109/TMC.2005.68

[15] J. Pan, Y. T. Hou, L. Cai, Y. Shi and S. X. Shen, "Topology Control for Wireless Sensor Networks,” Proceedings of ACM International Conference on Mobile Computing and Networking (MobiCom), September 2003, pp. 286299.

[16] T. S. Rappaport, "Wireless Communications: Principles \& Practice,” Prentice-Hall, New Jersey, 1996.

[17] G. Gupta and M. Younis, "Fault-Tolerant Clustering of Wireless Sensor Networks," Proceedings of IEEE Wireless Communications and Networking Conference (WCNC), Vol. 3, 2003, pp. 1579-1584.

[18] W. Heinzelman, A. Chandrakasan and H. Balakrishnan, "Energy-Efficient Communication Protocol for Wireless Microsensor Networks," Proceedings of Hawaiian International Conference on Systems Science, 4-7 January 2000, pp. 1-10. doi:10.1109/HICSS.2000.926982

[19] O. Younis and S. Fahmy, "HEED: A Hybrid, EnergyEfficient, Distributed Clustering Approach for Ad Hoc Sensor Networks,” IEEE Transactions on Mobile Computing, Vol. 3, No. 4, 2004, pp. 366-379. doi:10.1109/TMC.2004.41

[20] Y.-B. Ko and N. H. Vaidya, "Location-Aided Routing (LAR) in Mobile Ad Hoc Networks,” Wireless Networks, Vol. 6, No. 4, 2000, pp. 307-321. doi:10.1023/A:1019106118419

[21] I. Stojmenovic and X. Lin, "Power-Aware Localized Routing in Wireless Networks," IEEE Transactions on Parallel and Distributed Systems, Vol. 12, No. 11, 2001, pp. 1122-1133. doi:10.1109/71.969123 